\title{
Estudio descriptivo sobre el juego patológico en estudiantes (8-17 años): Características sociodemográficas, consumo de drogas y depresión
}

\author{
Arbinaga, F. \\ Psicólogo
}

Enviar correspondencia a:

Félix Arbinaga Ibarzábal. Centro de Psicología Clínica. C/. José Fariña, 56 - 5 c. Huelva 21006. Tel. 959251488

\begin{abstract}
RESUMEN
Se presentan los resultados obtenidos en un grupo de menores (8-17 años) dentro de un estudio epidemiológico sobre el juego de apuesta y el juego patológico realizado en el municipio de Punta Umbría (Huelva). Los datos se recogieron sobre una muestra de 130 sujetos, utilizando el South Oaks Gambling Screen (SOGS-RA) para adolescentes, donde podemos observar que el 83,1\% no presentaría problemas de juego, el 11,5\% sería jugador con problemas leves y el $5,4 \%$ podría considerarse como probable jugador patológico. Tras describir distintas características sociodemográficas, ofrecemos información sobre frecuencia y uso de drogas en los jugadores. Destacamos que el 14,3\% de los jugadores patológicos fuman diariamente, dándose igual porcentaje para el consumo de alcohol. Por otra parte, el $71,5 \%$ de los jugadores patológicos superan los 17 puntos en el CDI, llegándose al 42,9\% que tendría pensamientos de suicidio, aunque no llegaría a realizarlo
\end{abstract}

Palabras Claves: Juego patológico, Menores de edad, Uso de drogas, Abuso de Alcohol.

\section{INTRODUCCION}

$\mathbf{E}$ stá bien documentado que, desde 1936, el juego ha experimentado un continuo y rápido crecimiento en la mayoría de los países (Frey, Eadington, 1984; McMillen, 1996). Una causa para este crecimiento que ha sido compartida por diversos autores es la disponibilidad de los juegos (Cornish, 1978; Connors, 1983; Dickerson, 1984; Orford, 1985). Sin embargo, la afirmación de que el rápido crecimiento del juego es atribuible a la legalización y a la accesibilidad al juego, puede ser considerado al menos incompleto; ya que toda actuación sería inútil si no hubiera un deseo de jugar por parte de la gente. Incluso en las

\section{SUMMARY}

This paper is a presentation of the results which were obtained from an epidemical study on gambling and pathological gambling carried out in the town of Punta Umbría (Huelva-Spain). It shows the data collected from a sample of 130 subjects who are under age (8-17 age), using the South Oaks Gambling Screen (SOGS-RA) for adolescents, where it can be observed that $83,1 \%$ do not present any gambling problems, $11,5 \%$ are would be gamblers with slight problems and $5,4 \%$ could be classified as probable pathological gamblers. After describing different sociodemographic characteristics, we offer information about the frequency and use of drugs in the gamblers. It can be noted that $14,3 \%$ of the gamblers are daily smokers, with the same percentage consuming alcohol. On the other hand, $71,5 \%$ of gamblers go over the 17 points on the CDI, and $42,9 \%$ could think of committing suicide, although they would not reach the point of doing so.

Key words: Pathological gambling, children under age, Use of drugs, Abuse of Alcohol.

sociedades donde el juego ha sido prohibido éste ha florecido (Dixon, 1996).

Para Ladoucer y Walker (1998) la naturaleza esencial del juego es que el dinero (o su equivalente) se arriesga en el incierto resultado de un suceso, sujeto a ciertas condiciones: a) el juego ocurre en un contexto de grupo donde después de los costos, tasas y ganancias, el dinero apostado por los perdedores es redistribuido a los ganadores y b) la redistribución del dinero es independiente de cualquier otra empresa comercial relacionada con el suceso de jugar.

El juego patológico fue reconocido oficialmente como entidad nosológica de salud mental en el año 1980 cuando la Sociedad Americana de Psiquiatría lo

Este trabajo ha sido financiado por la Concejalía de Bienestar Social (Ayto. de Punta Umbría, Huelva). 
incluye por primera vez como trastorno en el Manual Diagnóstico y Estadístico de los Trastornos Mentales, en su tercera edición (D.S.M.III) (APA, 1980). En ella, los criterios diagnósticos del juego patológico aparecen reflejados entre los trastornos de control de impulsos no clasificados en otras categorías o trastornos residuales. El rasgo básico que caracteriza al jugador patológico es "un fracaso crónico y progresivo en la capacidad de resistir los impulsos a jugar y de la conducta de jugar, fracaso que compromete, altera o lesiona los intereses personales, familiares y vocacionales" (APA, 1987, 388). En 1987, cuando se publica la revisión del Manual (D.S.M.III-R) se producen cambios importantes en la consideración que del juego patológico se tiene, centrando el interés en aspectos como los síntomas fisiológicos de la abstinencia y tolerancia. Por su parte, la cuarta versión del DSM (APA, 1995) mantiene que la característica esencial del juego patológico "es un comportamiento de juego, desadaptativo, persistente y recurrente" y se han de cumplir al menos cinco de los criterios indicados en la Tabla 1; así mismo reflejamos la propuesta de Fisher (1992) para ajustar los criterios en niños de entre 11 y 16 años (DSM IV-J).

\section{Tabla 1. Criterios diagnósticos del juego patológico en el D.S.M.IV. y DSM IV-J.}

\section{DSM IV}

1. Preocupación por el juego.

2. Necesidad de jugar con cantidades crecientes de dinero para conseguir el grado de excitación deseado.

3. Fracaso repetido en el esfuerzo de para controlar, interrumpir o detener el juego.

4. Inquietud o irritabilidad cuando intenta interrumpir o detener el juego.

5. El juego se utiliza como estrategia para escapar de los problemas.

6. Después de pérdidas se regresa para recuperar.

7. Se engaña a los miembros de la familia y otros para ocultar el grado de implicación con el juego.

8. Se comenten actos ilegales para financiar el juego.

9. Se han arriesgado o perdido relaciones interpersonales, trabajos etc.

10. Se confía en que los demás proporcionen dinero que alivie la desesperada situación financiera.

Las definiciones dadas en las distintas versiones del DSM asumen que en la población existen, al menos, dos tipos de jugadores: los sociales y los patológicos. Mientras los primeros serían aquellos que juegan esporádicamente y tienen control sobre su conducta, los jugadores patológicos se caracterizarían por jugar frecuentemente y sin control de su conducta de juego. Shaffer, Hall, Vander (1997) agrupan a los jugadores en tres niveles, que bien pudieran representar un continuo de incremento en las conductas de jugar y los problemas asociados. Nivel 1.- juego social o recreacional sin consecuencias significativas. Nivel 2.- juego con problemas moderados y Nivel 3.conductas de juego que resultan en múltiples problemas y es consistente con los criterios del DSM. Por su parte, Ochoa y Labrador (1994) proponen cuatro grupos de jugadores: los jugadores sociales, los jugadores profesionales, los jugadores problemas y juga-

\section{DSM IV-J}

1. Preocupación por el juego.

2. Necesidad de jugar con cantidades crecientes de dinero para conseguir el grado de excitación deseado.

3. Fracaso repetido en el esfuerzo de para controlar, interrumpir o detener el juego.

4. Inquietud o irritabilidad cuando intenta interrumpir o detener el juego.

5. El juego se utiliza como estrategia para escapar de los problemas.

6. Después de pérdidas se regresa para recuperar.

7 Se engaña a los miembros de la familia o amigos para ocultar el grado de implicación en el juego.

8. Se cometen actos ilegales/antisociales, gastar dinero de la comida, robar en casa o fuera para jugar.

9. Se riñe con la familia o con los amigos más próximos y se hace peligrar la educación por el juego.

10. Se confía en que los demás proporcionen dinero que alivie la desesperada situación financiera.

dores patológicos (adicto y dependiente, respectivamente, en la terminología de Irurita, (1996).

Hoy en día hay que reconocer que aún no se dispone de datos concluyentes en el estudio de la personalidad de los jugadores, no hay una evidencia empírica ni teórica que permita caracterizar a éstos como grupo homogéneo (Fernández, Echeburúa, Báez, 1999). Sólo mencionar que los dos aspectos que más han llamado la atención en el estudio de la personalidad son el constructo de búsqueda de sensaciones (Zuckerman, 1979; Brown, 1993) y los trabajos sobre impulsividad (Castellani, Rugle, 1995; Steel, Blaszczynski, 1996; Fernández, Echeburúa, Báez, 1999). Por otra parte, y dada la alta incidencia de antecedentes familiares de ludopatía observada en muestras clínicas de jugadores patológicos (20\%-35\%, si se considera a familiares de primer grado y/o segundo 
grado respectivamente) se está desarrollando la investigación genética para determinar cuáles son los genes candidatos específicos del trastorno como así ocurre en otras adicciones (Cadoret, Yates, Troughton, Woodworth, Stewart, 1995; Saiz, Ibáñez, 1999).

Entre los rasgos clínicos se ha podido constatar que los jugadores patológicos obtienen puntuaciones superiores en depresión a las de la población control y una mayor presencia de acontecimientos vitales significativos y con episodios de depresión mayor si se evaluaban retrospectivamente (Roy, Ardinoff, Roehrich, Lamparski, Custer, Lorenz, 1988; McCormick, 1993; Becoña, 1993; Becoña, Lorenzo, Fuentes, 1996; Crockford, el-Guebaly, 1998). Al igual que con los estados afectivos, y relacionado con ellos, parece que se dan ciertas coincidencias entre actividades de juego y el suicidio (Phillips, Welty, Smith, 1997).

La ejecución de conductas de juego suele realizarse en contextos donde las posibilidades de acceder a ciertos tipos de sustancias (vg. alcohol, tabaco) son altas y reforzadas. En este sentido ha existido una cierta preocupación por conocer las relaciones que puedan darse entre el consumo de sustancias adictivas y el juego. Así Lesieur (1985) ya señalaba que el $15 \%$ de los jugadores patológicos también mostraban adicción al alcohol y/u otro tipo de drogas. En España la situación es similar, se observa como el $25 \%$ de los jugadores patológicos consumen 100cc o más de alcohol frente al $5 \%$ de la población general que lo hace en ese nivel. Rodríguez Martos (1987) encuentra que el $15 \%$ de las personas diagnosticadas como alcohólicas muestran un doble diagnóstico de juego patológico. En grupos de menores y con relación al uso de tabaco, el 16,3\% de los que decían consumir actualmente presentaban problemas con el juego y el $11 \%$ de los que reconocían beber (Arbinaga, 1996).

Pero junto a este tipo de alteraciones que sufren los jugadores podemos observar un conjunto de manifestaciones relacionadas con la abstinencia al juego. En los momentos de abandono de la actividad de jugar aparece un fuerte deseo de volver a jugar y un aumento de la irritabilidad (Wray, Dickerson, 1981). Sobre una muestra de 222 jugadores patológicos (Rosenthal, Lesieur, 1992) destaca que el $24 \%$ se considera alcohólico y el 18\% adicto a drogas. El 65\% de los jugadores ha padecido al menos uno de los siguientes síntomas de abstinencia: insomnio, dolor de cabeza, trastornos digestivos o diarrea, pérdida del apetito, debilidad física, palpitaciones, dificultad en respirar, temblor, calambres, sudoración, dolores musculares, fiebre y el $91 \%$ reconocía experimentar "craving" (ansia por jugar).

Las principales fuentes de información epidemiológica, en la literatura internacional, las encontramos en los trabajos procedentes de EEUU, Canadá, Australia y países avanzados de Europa, Reino Unido, Alemania, Francia (Gallup, 1976; Meyer, 1986; Dickerson,
Hinchy, 1988; Volberg, Steadman, 1988; Volberg, 1990; Ladoucer, 1991; Shaffer, Hall, 1996; Shaffer y cols., 1997; National Opinion Research Center, 1999; entre otros). Por parte de nuestro contexto más cercano, y al mismo nivel de análisis global, destacan diversas investigaciones sobre la incidencia del juego en las Comunidades Autónomas de Andalucia (Legarda; Babio, Abreu, 1992; Irurita, 1996; Tejeiro, 1998), Galicia (Becoña, Fuentes, 1995; Becoña, 1991) y en Cataluña (Cayuela, 1990).

En el ámbito internacional la tasa de prevalencia del juego patológico en la población adulta se sitúa entre el 2\%-3\%, según lo manifestado en el DSM-III$R$ (APA, 1987) y el 0,5\%-3\% según otros estudios realizados (Allcock, 1986; Volberg y cols. 1988). A partir del meta-análisis realizado por Shaffer, Hall y Bilt (1997), sobre 18 estudios llevados a cabo entre el año 1988 y 1997, en los Estados Unidos y Canadá, se encuentra que la prevalencia, a lo largo de la vida de jugadores patológicos (nivel 3), se mueve en el rango del $0,1 \%$ al 3,1\%, con una media de 1,5\%. La estimación combinada de los problemas de juego y la prevalencia de los probables patológicos (nivel 2 y 3 ) se sitúa entre el 2,3\% y el 12,9\%, éstos sobre 15 estudios, y con una media del 5,4\%.

En 1986, la Comisión Nacional del Juego publica en nuestro país "El Libro Blanco del Juego" donde se pone de manifiesto que el 79,2\% de los mayores de 16 años reconoce jugar en mayor o menor medida, perfilándose un jugador tipo. Estudios más recientes, desde el año 1990, nos mantienen en niveles de incidencia poblacional entre valores que oscilan, para jugadores problemas del 1,6\% (Becoña, 1991) al 5,2\% de Legarda y cols. (1992) y, para jugadores patológicos del 1,4\% encontrado por Becoña y Fuentes (1995) al 1,9\% de Tejeiro (1996).

En Andalucía, los datos de Irurita (1996) nos reflejan que el 1,8\% del conjunto de la población andaluza probablemente sea jugadora patológica frente al 4,4\% que mantendría problemas leves con el juego. Considerando los resultados obtenidos en las tasas por tipo de jugador para cada provincia destacan las zonas de Cádiz (11,5\%), Málaga (8,3\%) y Jaén (7,2\%). Por su parte, Huelva presentaría un 4,18\% en ambas categorías situándonos en la sexta provincia de nuestra comunidad.

Recientemente, en el panorama internacional se ha publicado nueva información sobre jóvenes, donde Westphal, Rush, Stevens y Johnson (2000) con una muestra de 11.736 estudiantes $\left(6^{\circ}-12^{\circ}\right.$ grado) reflejan que el $10,1 \%$ de los mismos indicaban problemas con el juego durante el año anterior y el 5,8\% podía presentar un juego patológico. Estos porcentajes podían aumentar considerablemente llegando hasta el $11 \%$ o el 19,9\% cuando se consideraban según los grados o cursos en los que se recogía la información. 
En nuestro país los estudios sobre menores de edad han comenzado a realizarse desde el año 1996 y los esquematizamos en la Tabla 2.
Hay que decir, que no es de extrañar que las tasas de juego en población menor de edad sean mucho más altas que las informadas en grupos de adultos; ya

Tabla 2. Estudios en España sobre el juego patológico en menores.

\begin{tabular}{lccccc}
\hline Autor & Año & Muestra & Edad & J. Patológico & Contexto \\
\hline Arbinaga & 1996 & 105 & $14-17$ & $2,9 \%$ & Huelva \\
\hline Becoña, Gestal & 1996 & 1.200 & $12-14$ & $2,2 \%$ & Galicia \\
\hline $\begin{array}{l}\text { Villa, Becoña } \\
\begin{array}{l}\text { Vázquez } \\
\hline \text { Castro }\end{array}\end{array}$ & 1997 & 2.185 & $11-16$ & $1,6 \%$ & Asturias \\
\hline Tomado y ampliado de Secades y Villa (1998). & 1999 & 153 & $15-17$ & $2,6 \%$ & I.Canarias \\
\hline
\end{tabular}

que viene siendo norma en muchos trabajos que las tasas de los adultos puedan incluso multiplicarse por cinco si el grupo estudiado está formado por jóvenes adolescentes (Lesieur, Blume, 1991; Ladoucer, Mireault, 1988, Fisher, 1992, Fisher, 1993, Westphal, Rush, Stevens, Johnson, 2000).

Revisando los datos informados sobre grupos de menores vemos que los varones juegan más que las mujeres (Arbinaga, 1996; Becoña, Gestal, 1996; Villa, Becoña, Vázquez, 1997). Así mismo, si se considera la edad, tanto Arbinaga (1996) como Villa, Becoña, Vázquez (1997) encuentran los porcentajes mas altos en los sujetos mayores de 13 años, mientras Becoña y Gestal (1996) señalan a esta edad como la más problemática dentro del grupo de jugadores patológicos con el $37 \%$ de los mismos.

De los estudios realizados en nuestro país sobre menores de edad sólo dos preguntaban por los antecedentes familiares relacionados con el juego (Becoña, Gestal, 1996; Villa, Becoña, Vázquez, 1997). En este sentido, los datos resultan contradictorios ya que la opinión mayoritaria de los jugadores patológicos a la pregunta que se les hace sobre si sus padres juegan a juegos de apuestas, el $48,1 \%$ y $38,2 \%$ dicen que sí, respectivamente en ambos estudios; aunque son pocos los que reconocen que alguno de ellos juega demasiado. Tampoco coinciden los trabajos cuando se trata de identificar al que juega demasiado; así, el $66,7 \%$ de los jugadores patológicos dicen que ambos juegan demasiado y un 33,3\% diría que es el padre (Becoña, Gestal, 1996) frente al trabajo de Villa y cols (1997) donde el $40 \%$ dice que son ambos los que juegan demasiado, otro $40 \%$ dice que es la madre y sólo el $20 \%$ dice que es el padre

Para finalizar queremos hacer mención al trabajo de Villoria (1999), que si bien no trabaja con menores de edad, aporta datos recogidos en muestras de universitarios sobre actitudes hacia el juego y hacia el jugador patológico, entre otros aspectos. En él se nos indica que se ha producido un cambio de actitud social respecto al consumo de drogas, especialmente con el alcohol; sin embargo, el juego patológico sigue sumergido en un prejuicio social con amplias repercusiones en la prevención y rehabilitación.

Decir, por último, que cuando se busca caracterizar al jugador en nuestro país (Becoña, 1999) los datos nos vienen a mostrar una realidad coincidente con la encontrada en otras zonas como los EEUU (National Opinion Research Center, 1999; Volberg, 1993) y Canadá (Ladoucer, 1991).

Partiendo de esta base, entre los objetivos que contemplábamos al inicio de este trabajo destacamos el aportar datos que permitan ampliar cuantitativa y cualitativamente la información sobre la adicción al juego en nuestro país. Conocer la prevalencia en el consumo de juegos de apuestas y los niveles de juego patológico entre los menores de edad del municipio de Punta Umbría (Huelva), como base para el ulterior diseño de protocolos de intervención, tanto preventivos como asistenciales.

\section{MÉTODO}

Por todo ello, dado el interés del estudio y el mantener un carácter de aproximación, utilizaremos un diseño descriptivo, localizando como criterio de clasificación, que caracterizará al grupo de referencia, los resultados al instrumento de evaluación sobre conductas relacionadas con el juego de apuesta.

Los datos que presentamos referentes a los menores de edad, se enmarcan en un estudio más amplio sobre el conjunto de la población del municipio de Punta Umbría (Huelva).

\section{Instrumentos}

Para llevar a cabo la recogida de información se confeccionó una hoja de respuestas específica. Ésta, en formato $\mathrm{A} 3$, se compuso de los siguientes bloques 
temáticos que a su vez se estructuraron basándose en diversos instrumentos (siempre se indicaba el período temporal de referencia):

\section{Bloque Primero. Datos de Sociodemografía. \\ Bloque Segundo. Datos de Escolares. \\ Bloque Tercero. Datos de Drogas y Tabaco. \\ Bloque Cuarto. Datos de Juego con apuesta. \\ Bloque Quinto. Datos de Motivación para el juego \\ Bloque Sexto. Datos de Depresión}

El primero de los bloques, aportaba información sobre datos sociales básicos del entrevistado: sexo, edad, número hermanos, lugar que ocupa, ingresos de los padres, gente con la que convive, etc, entre otras cuestiones. Aquí se decidió introducir una pregunta sobre si conocía el hecho de que alguna de las personas con las que convivía jugaba apostando, la intención fue la de unificar las preguntas sobre las persona con las que compartía la vivienda.

El segundo de los bloques se compuso de información sobre nivel de estudios, repeticiones, grado de satisfacción con los estudios y grado de apoyo de los padres en los estudios.

El bloque tercero (últimos seis meses) se compuso de una pregunta de opción múltiple sobre el uso de ocho tipos de sustancias (porros, alcohol, alucinógenos, tabaco, cocaína, heroína, anfetaminas, tranquilizantes) y la frecuencia de uso (nunca he probado, he probado pero ahora no consumo, actualmente varias veces al mes, actualmente varias veces a la semana, todos los días) y una sobre intensidad en el consumo de tabaco ( $n^{\circ}$ cigarrillos/día).

El cuarto de los bloques se lleva a cabo para valorar las conductas de juego y para ello se utiliza el SOGS adaptado a la población adolescente (SOGS-RA de Winters, Stinchfield y Fulkerson, 1993) dicha versión aún se encuentra en fase experimental y los autores no aportan información sobre su capacidad para identificar y clasificar correctamente a los jugadores patológicos adolescentes, aunque ya se comienzan a ver estudios con tal instrumento como medida del juego en la población adolescente (Westphal, Rush, Stevens, 1998), para ampliar información sobre la evaluación, a través de distintos instrumentos, del juego patológico en adolescentes remitimos al lector al trabajo realizado por Secades y Villa (1998).

Como decimos el SOGS-RA es una adaptación a la población adolescente del Cuestionario de Juego de South Oaks (SOGS) de Lesieur y Blume (1987). Instrumento de "screening" que identifica de manera rápida a posibles personas en dificultades con las conductas de jugar, se ha mostrado como un instrumento de elección prioritario en los diversos estudios realizados, tanto en nuestro país como internacionalmente, siendo considerado como un predictor de un criterio hete- rogéneo (DSM.III-R) para el juego patológico; además de ser el instrumento más fiable y válido de que se dispone en la actualidad. Se destaca su interés (Echeburúa, 1994) por permitir una detección más precoz en fases críticas del juego patológico. Entre sus limitaciones se menciona el ser un instrumento de aproximación a poblaciones de riesgo y no permitir análisis funcionales de la problemática individual. Culleton (1989) centra sus críticas al considerarlo como una medida que falla en explicar los falsos positivos cuando se usa una muestra de la población general como opuesto a los grupos de validación originales.

Para ampliar información sobre las características específicas del instrumento adaptado a la población española remitimos a Echeburúa, Báez, FernándezMontalvo y Páez (1994) y Echeburúa (1994). Y para revisar algunas dificultades en su uso puede consultarse a Dickerson (1985), Culleton (1989), Solonsch (1989), Dickerson (1993) y al National Research Council (1999).

El quinto de los bloques (doce últimos meses) se organizó en torno a seis preguntas sobre diversas motivaciones alegadas para jugar (para ganar dinero, para entretenerse, por que lo hacen los amigos, por las sensaciones obtenidas, para olvidar problemas y por que no puedo dejar de jugar cuando empiezo) dicho bloque de preguntas tenía como opciones de respuesta desde 1 (nunca) hasta 5 (siempre) y fue tomado de Irurita (1996).

El último de los bloques se compuso del Children`s Depression Inventory (C.D.I.) (Kovacs, Beck, 1977). Dicha prueba deriva del BDI (Beck, Rush, Shaw, Emery, 1979) al que se le reducen el número de alternativas, se le cambian varios ítems, se suprime el referido al interés sexual y se introducen otros relacionados con el colegio y se le simplifica el lenguaje. Por lo que se refiere a los datos psicométricos podemos decir que el rango de respuesta es de 0 a 54. Las categorías de clasificación utilizadas según la puntuación obtenida, sobre los 27 ítems de que consta el instrumento, parten de los siete puntos para los casos de depresión leve.

Los coeficientes alfa de Cronbach con muestras españolas varían de 0,79 (Frías, del Barrio y Mestre, 1991) a 0,92 (Polaino-Lorente, García-Villamisar, 1993) y con muestras extranjeras de 0,80 a 0,94 (Saylor, Finch, Spirito y Benet, 1984) mostrando consistencia interna satisfactoria (Mendez, 1999). El rango de fiabilidad test-retest oscila de 0,38 a 0,87 (Saylor, Finch, Spirito y Benet, 1984).

Por lo que a la validez se refiere, la prueba discrimina a niños con y sin depresión (Lovobits, Handal, 1985). También correlaciona positivamente con otros autoinformes: 0,81 en Asarnow, Carlson (1985); 0,84 en Rotundo, Hensley (1985) y 0,44 en el trabajo de Weisman, Orvaschel, Padian (1980). 


\section{Muestra}

El estudio que presentamos ha sido realizado en el municipio de Punta Umbría, provincia de Huelva. Su ubicación dentro de la provincia, en la zona sur, centro de la costa, la convierten en una demarcación geográfica privilegiada para el turismo y el ocio. Junto al turismo, el municipio se caracteriza, en el conjunto de la provincia, por el desarrollo de una actividad productiva inmersa en el ámbito de la pesquería (Serna, 1996).

La provincia de Huelva en 1996, presentaba una población censal de 452.822 personas (Instituto Nacional de Estadística, 1996). El municipio de Punta Umbría, por su parte, reflejaba una población de 10.888 personas, situándose entre los diez términos municipales de mayor población de la provincia (ésta cuenta con 79 municipios), y perteneciendo a una de las zonas, la de costa, que resulta en una de las de mayor densidad en habitantes. En el censo se puede observar que el $49,1 \%$ son mujeres y el $79,1 \%$ es menor de 50 años. El intervalo de edad considerado para nuestros intereses (8-17 años) implica al 17,75\% de la población $(n=1.932)$ siendo el $50,3 \%$ hombres y el $49,7 \%$ mujeres.

Para la recogida de información se realizó un muestreo estratificado, proporcional aleatorio, considerando el criterio de clasificación la edad y el sexo. El error establecido para la determinación del tamaño muestral es de $\pm 4 \%(e=0,04)$ y un nivel de confianza del $95,5 \%$ (Tagliacarne, 1962), esto hace que el número de personas requeridas para caracterizar a la muestra para una población como la comentada sea de 588 sujetos. Una vez recogida la información, la muestra quedó compuesta por 616 individuos, con edades comprendidas entre los 8 y los 79 años. Nosotros presentaremos los datos que se obtuvieron en la población menor o igual a 17 años. Ésta se compuso de 130 personas (45,4\% mujer y 54,6\% varón).

\section{Procedimiento}

La recogida de información fue realizada por un total de cinco personas, cuatro estudiantes de los últimos cursos de psicología de la UNED y el coordinador, identificadas con una autorización municipal. Todos se sometieron a cinco sesiones de formación sobre: juego, instrumentos, metodología de trabajo, instrucciones etc.. Decir que para los análisis estadísticos se ha utilizado el SPSS-PC 6.0. El período durante el que se procedió a la recogida de la información fue el que medió entre el 2 de octubre y el 1 de noviembre de 1999. Las horas sobre las que se trabajaba eran las lectivas del colegio o instituto.

Para la recogida de la información se contó con la colaboración de la dirección de los centros escolares y los tutores. Seleccionados los niveles académicos, la representación por sexos y las clases donde se pedi- rían los voluntarios se acudía en horas de tutoría. Cuando se disponía de los sujetos, elegidos al azar entre los voluntarios, se les trasladaba a un aula preparada para el caso. Se les daba las instrucciones, unificadas para todos los grupos, insistiendo en el carácter anónimo, y se les mantenía separados para garantizar la confidencialidad de las respuestas. Se insistía en qué se consideraban juegos de apuesta y se mencionaban ejemplos. Con los sujetos de 8 a 11 años, se establecían grupos de tres a los que se dirigía un colaborador de manera específica, éste les leía cada una de las preguntas y el joven respondía tras la lectura de cada una de ellas. El colaborador solía aclarar cada una de las preguntas asegurándose que el joven entendiese las cuestiones. El colaborador no veía las respuestas dadas por los jóvenes, ni éstos las de sus compañeros.

\section{RESULTADOS}

Los resultados obtenidos en la evaluación de la prevalencia de las conductas de juego en el grupo de menores nos muestran que el $83,1 \%$ son sujetos que no tendrían problemas con el juego. El 11,5\% mantendría problemas leves con el juego y el 5,4\% podríamos clasificarlo con mucha probabilidad como jugador patológico. En la Tabla 3 resumimos las principales características sociodemográficas de la muestra. En ella podemos observar el fuerte incremento que se refleja en lo que representan las mujeres dentro de las categorías de problemas con el juego cuando se pasa del grupo de probable patología al de problemas leves.

Como podemos ver el $71,5 \%$ de los jugadores patológicos tienen entre 12 y 15 años, frente al $60 \%$ que representan dichas edades en el grupo de problemas leves. El intervalo de edad dominante entre los jugadores patológicos es el de 12-13 años (42,9\%); sin embargo, en los problemas leves la distribución es mayor y destacan los $14-15$ años $(46,7 \%)$ y puntúan en el grupo 6-17 años.

De manera resumida podemos decir que la principal motivación alegada para jugar por el grupo de jugadores patológicos, y unificando las respuestas en sólo dos categorías ("nunca he jugado por.." y "alguna vez he jugado por..."), es la de entretenerse $(85,7 \%)$, seguida por la de ganar dinero $(71,5 \%)$, a continuación por las sensaciones obtenidas con el juego $(57,1 \%)$, la influencia de los amigos se iguala a la de no poder dejar de jugar una vez que ha iniciado el juego $(28,6 \%)$, siendo la motivación menos alegada la de jugar para olvidar los problemas (14,3\%).

Si revisamos los resultados en función del tipo de juego $(n=10)$ que más se reconoce como jugado en el último año (nunca, menos de una vez al mes, mensualmente, semanalmente y diariamente) y los agru- 
Tabla 3. Distribución del tipo de jugador según características socioeducativas.

\begin{tabular}{|c|c|c|c|c|c|}
\hline$n=130$ & $\%$ & Total & Sin Problema & J.Problema & J.Patológico \\
\hline Sexo & & & 83,1 & 11,5 & 5,4 \\
\hline Varón & & 54,6 & 54,6 & 46,7 & 71,4 \\
\hline Mujer & & 45,4 & 45,4 & 53,3 & 28,6 \\
\hline \multicolumn{6}{|l|}{ Edad } \\
\hline 8-9 & & 16,2 & 18,5 & 6,7 & - \\
\hline $10-11$ & & 29,2 & 33,3 & - & 28,6 \\
\hline $12-13$ & & 22,3 & 22,2 & 13,3 & 42,9 \\
\hline $14-15$ & & 23,8 & 20,4 & 46,7 & 28,6 \\
\hline $16-17$ & & 8,5 & 5,6 & 33,3 & - \\
\hline \multicolumn{6}{|l|}{ Ingresos Padres. } \\
\hline$<60.000$ & & 10,0 & 15,2 & 6,7 & - \\
\hline $60.001-120.000$ & & 23,8 & 30,4 & 40,0 & 16,7 \\
\hline $120.001-250.000$ & & 28,5 & 34,2 & 40,0 & 66,7 \\
\hline $250.001-400.000$ & & 11,5 & 15,2 & 13,3 & 16,7 \\
\hline$>400.000$ & & 3,1 & 5,1 & - & - \\
\hline \multicolumn{6}{|l|}{ Nivel de estudios. } \\
\hline $4^{\circ}$ Primaria & & 14,6 & 16,7 & 6,7 & - \\
\hline $5^{\circ}$ Primaria & & 13,8 & 16,7 & - & - \\
\hline $6^{\circ}$ Primaria & & 18,5 & 20,4 & - & 28,6 \\
\hline $1^{\circ} \mathrm{ESO}$ & & 12,3 & 12,0 & - & 42,9 \\
\hline $2^{\circ} \mathrm{ESO}$ & & 16,2 & 15,7 & 20,0 & 14,3 \\
\hline $3^{\circ} \mathrm{ESO}$ & & 11,5 & 11,1 & 20,0 & - \\
\hline $4^{\circ} \mathrm{ESO}$ & & 13,1 & 7,4 & 53,3 & 14,3 \\
\hline \multicolumn{6}{|l|}{ Repeticiones } \\
\hline $\mathrm{Si}$ & & 24,6 & 23,1 & 46,7 & - \\
\hline No & & 75,4 & 76,9 & 53,3 & 100 \\
\hline \multicolumn{6}{|c|}{ Satisfacción Estudios } \\
\hline Muy Baja & & 3,8 & 1,9 & - & 42,9 \\
\hline Baja & & 5,4 & 6,5 & - & - \\
\hline Normal & & 31,5 & 26,9 & 66,7 & 28,6 \\
\hline Alta & & 24,6 & 25,0 & 26,7 & 14,3 \\
\hline Muy Alta & & 34,6 & 39,8 & 6,7 & 14.3 \\
\hline
\end{tabular}

pamos en sólo dos categorías (nunca y alguna vez) veríamos que en el conjunto de la muestra el juego que domina sería rascar cartones para obtener premios $(48,5 \%)$, seguido del bingo en el que reconocen su participación el 31,5\% y en las máquinas tragaperras el $22,3 \%$, las loterías o similares lo han practicado el $18,5 \%$ y por detrás tenemos los deportes de equipo $(17,7 \%)$, las cartas $(16,2 \%)$, los juegos de habilidad $(15,4 \%)$, los juegos de cara o cruz $(6,9 \%)$ y en último lugar las carreras de animales (1,5\%).

Si el tipo de juego lo observamos desde la óptica del grupo de jugadores patológicos veríamos que el $57,1 \%$ dice haber jugado a las cartas; por su parte, al bingo, las máquinas tragaperras, rascar cartones y a cara o cruz lo reconocen el 42,9\% del grupo, el 28,7\% dice haber jugado a las loterías, dados, deportes de equipo o juegos de habilidad y sólo el 14,3\% reconoce haber apostado a carreras de animales.
A la hora de intentar determinar posibles influencias sobre las conductas de juego en los jóvenes, desde el seno familiar o en el contexto del domicilio, podemos observar que el 85,7\% de los jóvenes jugadores patológicos reconoce que viven con alguien que juega apostando. Igual porcentaje reconoce que alguno de sus padres juega, y cuando se le pregunta quién es el que juega el 66,7\% de los jugadores con una probable patología dicen que lo hacen ambos progenitores. Si la cuestión la centramos en conocer cómo valoran las conductas de juego de los padres, el $28,6 \%$ de este grupo dice que alguno de sus padres juega o ha jugado demasiado; siendo reconocido el padre en el $50 \%$ de los casos y ambos en la otra mitad. La madre no se reconoce en ninguno de los dos grupos con posibles problemas.

Cuando a los sujetos les demandamos información sobre el uso de sustancias, podemos ver que de los 
130 jóvenes el 50,8\% reconoce que, en los últimos seis meses, ha probado alguna vez una o más drogas. Entre los jugadores patológicos el 71,4\% reconoce el uso de dos sustancias y el $28,6 \%$ de una sola. En el grupo de sujetos con problemas leves el $80 \%$ dice haber probado dos o más drogas para igual período temporal.

Concretando el tipo de sustancia que se reconoce como consumida (Tabla 4) observamos que entre los jugadores patológicos hay tres sustancias, que con una u otra frecuencia se consumen actualmente (alcohol, tabaco, porros) destacando los grupos de jugadores patológicos que actualmente consumen alcohol y tabaco. Queremos llamar la atención sobre ese $42,9 \%$ de jugadores patológicos que han consumido tranquilizantes, aunque ahora no lo hagan. En el grupo de jóvenes con problemas leves de juego destaca que el 73,3\% de los mismos consume alcohol en la actualidad y un $46,7 \%$ lo hace con el tabaco.

\begin{tabular}{|c|c|c|c|c|}
\hline \multicolumn{5}{|c|}{ Tabla 4.- Consumo de drogas y problemas de juego } \\
\hline$n=130$ & Total & Sin Problema & J.Problema & J.Patológico \\
\hline Total & & 83,1 & 11,5 & 5,4 \\
\hline \multicolumn{5}{|l|}{ Porros } \\
\hline Nunca & 90,8 & 93,5 & 66,7 & 100 \\
\hline Ahora no & 5,4 & 4,6 & 13,3 & - \\
\hline Actualmente & 3,8 & 1,8 & 20,0 & - \\
\hline \multicolumn{5}{|l|}{ Alcohol } \\
\hline Nunca & 52,3 & 60,2 & 6,7 & 28,6 \\
\hline Ahora no & 25,4 & 24,1 & 20,0 & 54,1 \\
\hline Actualmente & 22,3 & 15,7 & 73,3 & 14,3 \\
\hline \multicolumn{5}{|l|}{ Alucinógenos } \\
\hline Nunca & 98,5 & 100 & 86,7 & 100 \\
\hline Ahora no & 1,5 & - & 13,3 & - \\
\hline Actualmente & - & - & - & - \\
\hline \multicolumn{5}{|l|}{ Tabaco } \\
\hline Nunca & 73,8 & 82,4 & 26,7 & 42,9 \\
\hline Ahora no & 10,8 & 6,5 & 26,7 & 42,9 \\
\hline Actualmente & 15,3 & 11,1 & 46,7 & 14,3 \\
\hline \multicolumn{5}{|l|}{ Cocaína } \\
\hline Nunca & 99,2 & 100 & 93,3 & 100 \\
\hline Ahora no & 0,8 & - & 6,7 & - \\
\hline Actualmente & - & - & - & - \\
\hline \multicolumn{5}{|l|}{ Heroína } \\
\hline Nunca & 100 & 100 & 100 & 100 \\
\hline Ahora no & - & - & - & - \\
\hline Actualmente & - & - & - & - \\
\hline \multicolumn{5}{|l|}{ Anfetaminas } \\
\hline Nunca & 100 & 100 & 100 & 100 \\
\hline Ahora no & - & - & - & - \\
\hline Actualmente & - & - & - & - \\
\hline \multicolumn{5}{|l|}{ Tranquilizantes } \\
\hline Nunca & 90,8 & 93,5 & 86,7 & 57,1 \\
\hline Ahora no & 8,5 & 6,5 & 6,7 & 42,9 \\
\hline Actualmente & 0,8 & - & 6,7 & - \\
\hline
\end{tabular}

Como vemos, las principales sustancias entre los jugadores, al igual que en el resto de la población, son el tabaco y el alcohol. Si nos acercamos a la intensidad en el consumo de tabaco o cantidad de cigarrillos fumados en el día, los sujetos probables patológicos en un $28,6 \%$ dice fumar hasta 10 cigarrillos. Entre los jugadores con problemas leves este porcentaje queda en el $33,3 \%$ y un $13,3 \%$ de los mismos dicen llegar hasta los 20 cigarrillos al día.

Por último, haremos una breve referencia a los resultados obtenidos en el cuestionario utilizado para 
medir alguna alteración en el estado afectivo. En el conjunto de la muestra el 59,2\% presentaría una puntuación, que para los criterios del instrumento utilizado, sería de normal en síntomas de depresión y un $18,5 \%$ presentaría una sintomatología de este tipo entre moderada y severa. En los sujetos sin problemas de juego los porcentajes representarían el 71,3\% y el $6,5 \%$ respectivamente, aumentando considerablemente su peso para los grupos de problemas leves y una probable patología (Tabla 5).

Tabla 5. Distribución de los jugadores según resultados C.D.I. e ítem $\mathbf{n}^{\circ} 9$.

\begin{tabular}{|lcccc|}
\hline $\mathbf{n = 1 3 0}$ & Total & Sin Problema & J.Problema & J.Patológico \\
\hline Total & & 83,1 & 11,5 & 5,4 \\
\hline C.D.I & & & & \\
$\quad$ Normal & 59,2 & 71,3 & - & - \\
$\quad$ Leve & 22,3 & 22,2 & 20,0 & 28,6 \\
$\quad$ Moderada & 10,0 & 5,6 & 33,3 & 28,6 \\
$\quad$ Grave & 8,5 & 0,9 & 46,7 & 42,9 \\
\hline Item no 9 Suicidio & & & & 57,1 \\
$\quad$ No lo Pienso & 67,7 & 73,1 & 33,3 & 42,9 \\
$\quad$ Pienso No Haría & 28,5 & 22,2 & 66,7 & - \\
Quiero & 3,8 & 4,6 & - & \\
\hline
\end{tabular}

No queremos dejar sin constatar los resultados obtenidos en el ítem número 9 del C.D.I y que hace referencia al suicidio, donde se puede observar que un grupo importante de jugadores con problemas ha pensado en suicidarse, aunque dice que no llegaría a culminarlo.

\section{CONCLUSIONES}

Los datos nos reflejan que considerando el conjunto de la muestra $(n=130)$ el $83,1 \%$ no presentaría problemas con el juego, el $11,5 \%$ estaría formando el grupo de sujetos con problemas leves y en el 5,4\% habría que considerar la posibilidad de que estuviéramos ante un grupo de jugadores con una patología relacionada con el juego.

Estos datos, aunque puedan resultar altos, cuando observamos los aportados en otros estudios de nuestro país, vienen a coincidir con lo informado por algunos autores en el ámbito internacional tanto para grupos de adultos (Shaffer, Hall, 1996; Shaffer, Hall, Bilt, 1997) como para grupos de adolescentes (Westphal, Rush, Stevens, Johnson, 2000). Así, Shaffer y Hall (1996) basándose en un meta-análisis de los estudios realizados en ocho estados de los Estados Unidos y cuatro provincias del Canadá, sobre un total de 19.000 sujetos, estimaron la prevalencia de los problemas de juego en el $14,8 \%$ y una prevalencia del juego patológico del 5,8\%. Posteriormente, Shaffer, Hall y Bilt (1997) concretaron que el porcentaje representado por los niveles 2 y 3 del juego vendrían a situarse entre el $2,3 \%$ y el $12,9 \%$ y con una media del $5,4 \%$; y si sólo se consideraba el nivel 3 (probable patología) los porcentajes se moverían entre el $0,1 \%$ y el 3,1\%, con una media del 1,5\%.

Por su parte, Westphal, Rush, Stevens y Johnson (2000) sobre una muestra de 11.736 estudiantes desde $6^{\circ}$ grado al $12^{\circ}$ grado de las escuelas de Louisiana, durante los años 1996 y 1997, reflejaron que el $10,1 \%$ de los jóvenes indicaban problemas con el juego durante el año anterior y el 5,8\% podía presentar un juego patológico (con intervalos de confianza al $95 \%$ que giraban desde el $9,6 \%$ al $10,6 \%$ y entre el $5,3 \%$ al $6,1 \%$ respectivamente). Los porcentajes aumentaban cuando se consideraban según los grados o cursos en los que se recogía la información, llegándose al $11 \%$ en el grado $11^{\circ}$ y al $19,9 \%$ en los grados $7^{\circ}$ y $8^{\circ}$. Sin embargo, era de esperar que fueran mucho más altas las tasas en este grupo que en el de los adultos, ya que teniendo en cuenta las revisiones existentes en la literatura se nos indica que las tasas de los adultos pueden incluso multiplicarse por cinco en el caso de que estemos estudiando a jóvenes adolescentes (Lesieur, Blume, 1991; Ladoucer, Mireault, 1988, Fisher, 1992, Fisher, 1993, Westphal, Rush, Stevens, Johnson, 2000).

Por último, cuando comparamos nuestros datos con los recogidos en entornos más próximos pero en adultos (Legarda, Babio, Abreu, 1992, Irurita, 1996, Tejeiro, 1998) se observa que para la combinación de ambas categorías de problemas con el juego (problemas leves y probable patología) tendríamos a un 6,9\% de los sujetos muestreados en la ciudad de Sevilla, a un $5,7 \%$ de los sujetos en la ciudad de Algeciras y a un $6,2 \%$ en el conjunto de la comunidad de Andalucía. Estos datos globales aumentan considerablemente en 
el trabajo de Irurita (1996) si revisamos la información de las dos categorías de problemas con el juego unificadas para las ciudades de Cádiz (11,5\%), Málaga $(8,3 \%)$ y Jaén $(7,2 \%)$.

Esto nos hace pensar que nuestros datos aun siendo altos se mantendrían dentro de las franjas superiores de la información que se nos ha reportado en trabajos de nuestro entorno cultural, tanto a nivel internacional y para grupos específicos, como en adultos de nuestra comunidad; aunque de esta última no dispongamos de datos sobre grupos de menores.

Realizando un breve repaso a los datos obtenidos vemos que los hombres presentan mayores problemas con el juego, sin embargo las mujeres muestran mayor peso en el grupo de los problemas leves, a diferencia de los trabajos ya mencionados (Arbinaga, 1996; Becoña, Gestal, 1996; Villa, Becoña, Vázquez, 1997).

Si observamos el nivel académico, el grupo que más peso tiene en los jugadores patológicos es el de $1^{\circ}$ de ESO y de $4^{\circ}$ ESO en los problemas leves. Así mismo, casi la mitad de los jugadores patológicos muestran una satisfacción muy baja con los estudios que realizan.

En cuanto al motivo alegado destacaríamos que el $57,1 \%$ de los jugadores patológicos reconoce que alguna vez ha jugado por las sensaciones obtenidas durante el juego y un 28,6\% por que no puede dejar de jugar una vez que ha comenzado la actividad. Si revisamos los tipos de juego en el grupo con más problemas, vemos que es frecuente jugar a actividades no permitidas a los menores; que si bien para algunas (cartas, bingo...) hay la posibilidad de desarrollarse en domicilios privados, y por tanto puede existir un menor control, otras han de hacerse en locales públicos (máquinas tragaperras).

El 71,5\% de los jugadores patológicos superan los 17 puntos en el CDI, llegándose al 42,9\% que tendría pensamientos de suicidio, aunque no llegaría a realizarlo. Si hablamos del consumo de drogas entre los jugadores patológicos el 14,3\% dice consumir de manera diaria tabaco y/o alcohol; porcentajes que aumentan considerablemente en lo que representan dentro del grupo de jugadores con problemas leves, situándose por encima de la media poblacional y similares a los encontrados por Arbinaga (1996).

A la vista de los resultados encontrados pensamos en la necesaria atención que deben recibir problemas como el que tratamos en municipios pequeños o medianos, donde, por sus peculiares características, la oferta de juegos es amplia. Así mismo veríamos conveniente el diseño de protocolos de actuación dirigidos a grupos específicos, que permita una asistencia global y generalizada a todo el espectro de patologías que pudieran asociarse al juego. Prestando especial atención a la evaluación de éste en los centros específicos de asistencia a drogodependientes.

Un problema como el juego patológico, donde la incidencia en la adolescencia es relevante, ha de entenderse en un proceso temporal, que actualmente es creciente. Sólo así se podrá comprender la importancia de las medidas dirigidas a objetivos de prevención primaria; teniendo como lugar de referencia el centro escolar. Para todo ello se hacen necesario procesos de evaluación que permitan conocer la evolución temporal del fenómeno, la eficacia y eficiencia de las medidas adoptadas.

\section{BIBLIOGRAFÍA}

Allcock,C.C.(1986): Pathological Gambling. Australian and New Zealand Journal of Psychiatry. 20,259265.

American Psychiatric Association (1980): Diagnostic and Stadistical Manual of Mental Disorders. (3rd.ed.).Washintong DC.APA.

American Psychiatric Association (1987): Diagnostic and Stadistical Manual of Mental Disorders. (3rd.ed.R).Washintong DC.APA.

American Psychiatric Association (1995): Diagnostic and Stadistical Manual of Mental Disorders. (IV).Washintong DC.APA.

Arbinaga, F. (1996): El juego patológico en estudiantes menores de 18 años: incidencia, uso de drogas y variables asociadas Adicciones. 8,3,331-348.

Asarnow, J.R.; Carlson, G. (1985): The depression selfrating scale: Utility with child psychiatric inpatients. Journal of Consulting and Clinical Psychology. 53, 491-499.

Beck, A.T.; Rush, A.J; Shaw, B.F.; Emery, G. (1979): Cognitive Therapy of Depression. N.Y. Guilford Press.

Becoña,E. (1991): The prevalence of pathological gambling in Galicia (Spain). Paper presented at the Meeting of the Society for the Study of Gambling. Londres.

Becoña, E.(1993): The prevalence of pathological gambling in Galicia (Spain). Journal of Gambling Studies. 9,353-369.

Becoña E (1999): Psicopatología y tratamiento de las adicciones. Fundación Universidad Empresa UNED.

Becoña, E; Gestal, C (1996): El juego patológico en niños del $2^{\circ}$ ciclo de EGB. Psicothema. 8, 13-23.

Becoña, E; Fuentes, MJ. (1995): El juego patológico evaluado con el South Oaks Gambling Screen. Adicciones. 7, 423-440. 
Becoña, E; Lorenzo, MC; Fuentes, MJ (1996): Pathological gambling and depression. Psychological Reports, 78, 635-640.

Brown RIF. (1993): El papel de la activación, distorsiones cognitivas y búsqueda de sensaciones en las adicciones al juego. Psicología Conductual. 1 375-388.

Cadoret RJ; Yates WR; Troughton E; Woodworth G; Stewart MA (1995): Adoption study demonstrating two genetic pathways to drug abuse. Archive General of Psychiatry 52, 1, 42-52.

Cayuela,R.(1990): Characteristics and Situation of Gambling Addiction in Spain: Epidemiological and Clinical Aspects. Eight International Conference on Risk and Gambling.Londres.

Castellani, B; Rugle, L (1995): A comparison of pathological gamblers to alcoholics and cocaine misusers on impulsivity, sensation seeking and craving. The International Journal of Addictions, 30, 275-289.

Castro J. (1999): Estudio de la proporción de jugadores patológicos en un grupo de menores, mediante el South Oaks Gambling Screen. El problema de los falsos positivos. Psicología.com Online 3, 1 http://www.psiquiatría.com/ psicología/vol3num1.

Comisión Nacional del Juego,(1986): Libro Blanco del Juego. Madrid. Ministerio del Interior.

Connors, X (1983): Report of the inquiry into a casino for Victoria. Melbourne: Victorian Governement Printer.

Cornish DB: (1978): Gambling: A review of the literature and its implication for policy and research. London HMSO.

Crockford DN; el-Guebaly N, (1998): Psychiatic comorbidity in pathological gambling: a critical review . Canadian Journal of Psychiatry. 43,43-50.

Culleton,R.P.,(1989):The prevalence rates of pathological gambling: A look at methods.Journal of Gambling Behavior.5,22-41.

Dickerson MG (1984): Compulsive Gamblers. London Longman.

Dickerson, MG (1985): The characteristics of the compulsive gambler; a rejection of a typology. En G. Cadwell, MG Dickerson, B Haig, L Sylvian (eds): Gambling in Australia. Sydney: Croom Helm.

Dickerson,M.G.(1993): Aproximaciones alternativas a la medición de la prevalencia del juego patológico. Psicología Conductual.1,3,339-349.

Dickerson,M.G., Hinchy,J.(1988): The prevalence of pathological gambling in Australia. Journal of Gambling Studies.4,135-141.

Dixon,D: (1996): Illegal betting in Britain and Australia: Contrasts in control strategies and cultures. In McMillen J: (ed): Gambling cultures; Studies in history and interpretation pp. 86-100. London Routledge.

Echeburúa,E.(1994): Evaluación y Tratamiento de los Trastornos Adictivos. Madrid. Fundación Universidad-Empresa.

Echeburúa, E; Báez, C; Fernández-Montalvo, J; Páez D.(1994): Cuestionario de juego patológico de South Oaks (SOGS): Validación española. Análisis y Modificación de Conducta. 20, 74, 769-793.

Fernández, JF; Echeburúa, E; Báez, C. (1999): Variables de inteligencia y de personalidad en los jugadores patológicos de máquinas tragaperras: un estudio descriptivo. Psicología Conductual. 7,3,349-360.

Fisher,S.E.(1992): Measuring pathological gambling in children: the case offruit machines in the U.K. Journal of Gambling Studies.8,263-285.

Fisher SE (1993): El impacto del juego de máquinas tragaperras legales para niños sobre el juego y juego patológico en adolescentes. El caso del Reino Unido. Psicología Conductual. 1, 351-359.

Frías, D; Del Barrio, V.; Mestre V (1991): Children's Depression Inventory. Evaluación Psicológical Psychological Assessment. 7, 377-391.

Frey JH.; Eadington WR. (1984): Gambling: Views from the social sciences. The Annals of the American Academy of Political and Social Science.474 (S.I).

Gallup, (1976): Gambling in Britain. Londres. HMSO. Social Surveys (Gallup Pool)

Instituto Nacional de Estadística (1996): Censo Poblacional del año 1.996.

Irurita,I.(1996): Estudio Sobre la Prevalencia de los Jugadores de Azar en Andalucía. Junta de Andalucía-FAJER Sevilla

Kovacs, M; Beck,A.T; (1977): An empirical-clinical approach toward a definition of childhood depression. En J.G. Schulterbarandt, A. Raskin (eds): Depression in childhood: Diagnosis, treatment and conceptual models. N.Y. Raven Press.

Ladoucer,R.(1991): Prevalence estimates of pathological gamblers in Québec, Canadá. Canadian Journal of Psychology.36,732-734.

Ladoucer,R., Mireault,C.(1988): Gambling behaviors among high school students in the Quebec area. Journal of Gambling Behavior.4,3-12.

Ladoucer, R; Walker;M.: (1998): Aproximación cognitiva para la comprensión y tratamiento del juego patológico. Revista de Psicología Contemporánea 5 (1). Pp 56-71

Legarda,J.J.,Babio,R.,Abreu,J.M.(1992): Prevalence estimates of pathological gambling in Seville (Spain). British Journal of Addictions. 87, 767770. 
Lesieur,H.R.(1985): The Chase. Career of the Compulsive Gambler. Cambridge, MA. Schenkman Books.

Lesieur,H.R., Blume,S.B.,(1987):The South Oaks Gambling Screen (SOGS): A new instrument for the identification of pathological gamblers. American Journal of Psychiatry. 144,1184-1188.

Lesieur,H.R.,Blume,S.B.,(1991): Evaluation of patients treated for pathological gambling in a combined alcohol, substance abuse and pathological gambling treatmenbt unit using the Addiction Severity Index. British Journal of Addictions, 86, 1017-1028.

Lovobits D.A; Handal, P.J. (1985): Childhood depression prevalence using DSM III criteria and validity of parent and child depression scales. Journal of Pediatric Psychology. 10, 45-54.

Meyer,G.(1986): Addiction to gambling. Zeistschrift fur Klinische Psychologie 34,140-156.

McCormick,RA (1993): Disinhibition and negative affectivity in substance abusers with and without a gambling problem. Addictive Behavior. 18,331336.

McMillen J. (1996): Gambling Cultures London Routledge.

National Opinion Research Center (1999): Overview of the National Survey and Community Database Research on Gambling Bahavior: Report to the National Gambling Impact Study Commission. Chicago IL

National Research Council (1999): Pathological Gambling: A critical review. Washington.

Ochoa,E. Labrador,F.(1994): El Juego Patológico. Plaza \& Janes.

Phillips, D; Welty, W; Smith, M (1997): Elevated suicide levels associated with legalized gambling. Suicide \& Life-Threatening Behavior. December 15.

Polaino-Lorente, A; García-Villamisar D. (1993): La depresión infantil en Madrid. Editorial AC. Madrid.

Rodríguez Martos,A.(1986): El diagnóstico del alcoholismo a través de cuestionarios Fons Informatiu. 2 julio 1986.

Rodríguez Martos,A.(1987): Estudio piloto estimativo de la prevalencia del juego patológico entre los pacientes alcohólicos que acuden al programa Dross. Revista Española de Drogodependencias. $14,265-275$

Rosenthal,RJ; Lesieur, H.R (1992): Self-reported withdrawal symptoms and pathological gambling. The American Journal of Addiction 1, 150-154.
Rotundo, N; Hensley, V.R. (1985): The Children's Depression Scale: A study of its validity. Journal of Child Psychology and Psychiatry. 26, 917-927.

Roy, A.; Ardinof, B.; Roehrich, L.; Lamparski, D.; Custer R.; Lorenz V. (1988): Pathological gambling: a psychobiological study. Archive General of Psychiatry 38, 108-112.

Saiz J; Ibañez, A (1999): Etiopatogenia neuroquímica de la ludopatía. Psiquiatría.com (revista electrónica) Marzo. 3 (1). http://www.psiquiatría.com/psiquiatría/ vol3num1/art_7,htm.

Saylor, C.F.; Finch, A.J.; Spirito, A; Benet, B. (1984): The Children's Depression Inventory: A systematic evaluation of psychometric properties. Journal of Consulting and Clinical Psychology. 52, 955-967.

Secades, R; Villa, A.. (1998): El juego patológico: prevención, evaluación y tratamiento en la adolescencia. Pirámide.Madrid.

Serna, S. (1996): Punta Umbría. Huelva y sus Pueblos. 63.1005-1018. Huelva Información.

Steel, Z; Blaszczynski, A. (1996): The factorial structure of pathological gambling. Journal of Gambling Studies. 12, 3-20.

Shaffer, HJ; Hall, MN,(1996): Estimating the prevalence of adolescent gambling disorders: a quantitative synthesis and guide toward standard gambling nomenclature. Journal of Gambling Studies.12, 193-214.

Shaffer, HJ; Hall, MN, Bilt J. (1997): Estimating the prevalence of disordered gambling behavior in the United State and Canada: A meta-analysis. Harvard Medical School Division on Addiction. December.10, 1997.

Solonsch, M (1989): The conceptualisation and evaluation of the concept of skill in gambling, Unpublished Honours Thesis, Australian National University, Camberra.

Tagliacarne, G. (1962): Técnicas y práctica de las investigaciones de mercado. Barcelona, Artiel.

Tejeiro, R (1996): Estudio sobre el juego de azar en Algeciras: Prevalencia y características sociodemográficas. Ayuntamiento de Algeciras.

Tejeiro, R (1998): El juego de azar en Algeciras (España): prevalencia y características sociodemográficas. Revista Española de Drogodependencias. 23,1,53-74

Villa, A Becoña, E; Vázquez, FL (1997): Juego patológico con máquinas tragaperras en una muestra de escolares de Gijón Adicciones. 9,195-208.

Villoria, C. (1999): Estudio piloto sobre la percepción social de los juegos de azar entre los universitarios: factores ambientales en el juego patológico. PsicoIogía.com Online 3, 1 http://www.psiquiatría.com/ psicología/vol3 n1 
Volberg,R.A.(1990): Estimating the Prevalence of Pathological Gambling in the United State. Eight International Conference on Risk and Gambling, agosto.

Volberg R (1993): Estimating the prevalence of pathological gambling in the United States. En WR Eadington, JA Corelius (eds): Gambling behavior and problem gambling. Reno NE. Institute for the Study of Gambling and Commercaial Gaming.

Volberg,R.A., Steadman,H.J.(1988): Refining prevalence estimates of pathological gambling. American Journal of Psychiatry.145,502-505.

Westphal, J.R.; Rush, J.A; Stevens, L. (1998): Problem and Pathological Gambling Behaviors within Specific Populations in the State of Indiana. (Gambling Behav ior of Indiana Students in Grades Six through Twelve 1997-1998 School
Year). Dpt. of Psychiatry Louisiana State University Medical Center-Sheveport, Louisiana.

Westphal, J.R.; Rush, J.A; Stevens, L.;Johnson,L. (2000): Gambling behavior of Louisiana students in grades 6 through 12. Psychiatric Services. 51, 1, 96-99. January.

Winters K.C.; Stinchfield R.D; Fulkerson J (1993): Toward the development of an adolescent gambling problem severity scale Journal of Gambling Studies 9, 63-84.

Wray, I; Dickerson, M (1981): Cessation of high frequency gambling and withdrawal symptoms. British Journal of Addictions 76, 401-405.

Zuckerman M. (1979): Sensation seeking: beyond the optimal level of arousal. Hillsdale NJ Lawrence Erlbaum. 
